\title{
金属过渡层类型对非晶碳膜结构性能的影响
}

\author{
李 蕾 ${ }^{1,2}$, 郭 鹏 $^{1}$, 刘林林 ${ }^{1}$, 孙丽丽 ${ }^{1}$, 柯培玲 ${ }^{1}$, 汪爱英 ${ }^{1}$
}

(1. 中国科学院宁波材料技术与工程研究所, 中国科学院海洋新材料与应用技术重点实验室, 浙江省海洋材料与 防护技术重点实验室, 宁波 315201; 2. 中国科学院大学, 北京 100049)

摘 要: 采用直流磁控溅射技术制备不同金属过渡层(Cr, Ti, W) 的类石墨非晶碳膜(GLC), 研究过渡层类型对非晶 碳膜微结构的影响, 并考察其在人工海水中摩擦性能的变化。研究结果表明: Cr/GLC 薄膜 $\mathrm{sp}^{2}$ 杂化键含量最高, 沿 GLC 表面到铬碳界面方向, $\mathrm{sp}^{2}$ 杂化键含量逐渐增大, $\mathrm{Ti}$ 过渡层和 $\mathrm{W}$ 过渡层的 $\mathrm{sp}^{2}$ 杂化键含量变化不明显。Cr/GLC 薄膜较高的 $\mathrm{sp}^{2}$ 杂化键含量有助于其在摩擦过程中产生可以充当润滑相的石墨化转化摩擦转移膜。在三种涂层中, $\mathrm{Cr} / \mathrm{GLC}$ 薄膜表现出最高的腐蚀电位 $-0.16 \mathrm{~V}$ 和最低的腐蚀电流密度 $4.42 \times 10^{-9} \mathrm{~A} / \mathrm{cm}^{2}$ 。因此相较于 $\mathrm{Ti}, \mathrm{W}$ 作过渡层 的 GLC 薄膜, Cr/GLC 薄膜在海水环境下表现出优异的摩擦学特性。

关 键 词: 海水; 类石墨非晶碳膜(GLC); 金属过渡层; 电子能量损失谱(EELS)

中图分类号: TH117 文献标识码: A

\section{Metal Buffer Layer on Structure, Mechanical and Tribological Property of GLC films}

\author{
LI Lei ${ }^{1,2}$, GUO Peng ${ }^{1}$, LIU Lin-Lin ${ }^{1}$, SUN Li-Li ${ }^{1}$, KE Pei-Ling ${ }^{1}$, WANG Ai-Ying ${ }^{1}$
}

\begin{abstract}
(1. Key Laboratory of Marine Materials and Related Technologies, Zhejiang Key Laboratory of Marine Materials and Protective Technologies, Ningbo Institute of Materials Technology and Engineering, Chinese Academy of Sciences, Ningbo 315201, China; 2. University of Chinese Academy of Sciences, Beijing 100049, China)
\end{abstract}

\begin{abstract}
Graphite-like carbon films (GLC) with different metal buffer layers (Cr, Ti, W) were fabricated by direct current magnetron sputtering technique. The effect of metal buffer layer on structure and tribological properties in artificial seawater were carefully investigated. The results show that, in contrast to Ti/GLC and W/GLC film, Cr/GLC film has the highest $\mathrm{sp}^{2}$ hybridization content, which increases from the top layer to $\mathrm{Cr} / \mathrm{C}$ interface. The highest $\mathrm{sp}^{2}$ hybridization content promotes graphitization degree of the tribo-film which acts as lubrication phase in the sliding process. The results of potentiodynamic polarization tests show that, in all of three GLC films, Cr/GLC film shows superior corrosion resistance with the highest corrosion voltage $\left(E_{\text {corr }}\right)$ of $-0.16 \mathrm{~V}$ and the lowest corrosion current $\left(i_{\text {corr }}\right)$ of $4.42 \times 10^{-9} \mathrm{~A} / \mathrm{cm}^{2}$. As a result, $\mathrm{Cr} / \mathrm{GLC}$ film presents the best tribological properties in artificial seawater in contrast to Ti/GLC and W/GLC films.
\end{abstract}

Key words: seawater; GLC; metal buffer layer; EELS

在过去的几十年中, 海洋经济在全世界飞速发展, 对于环境友好型和能源节约型新材料的需求与日俱
增。在海洋环境下, 海洋设备关键部件, 如水泵、气缸 活塞、轴承和船用螺旋桨等, 承受着机械摩擦和海水

收稿日期: 2017-05-03; 收到修改稿日期：2017-06-23

基金项目: 国家自然科学基金(51522106, 51375475); 浙江省公益项目(2016C31121), 宁波市自然科学基金(2015A610089) National Natural Science Foundation of China (51522106, 51375475); Pubic Project of Zhejiang Province (2016C31121); Ningbo Municipal Natural Science Foundation (2015A610089)

作者简介: 李 蕾(1991-), 女, 博士研究生. E-mail: llei@nimte.ac.cn

通讯作者: 汪爱英, 研究员. E-mail: aywang@nimte.ac.cn; 柯培玲, 研究员. E-mail: kepl@nimte.ac.cn 
腐蚀的双重损害作用, 关键部件在海洋环境中的摩擦 学性能直接决定着海洋工程装备的安全性能和服役时 间, 因此提高海洋工程装备中关键部件的综合性能刻 不容缓 ${ }^{[1]}$ 。研究者们采用诸多先进的技术方法 ${ }^{[2-3]}$ 增强 摩擦部件的综合性能, 从而提高海洋工程装备的安全 性、稳定性以及寿命。在众多先进的工程技术中, 制 备具有优异润滑性和耐蚀性的功能涂层是最经济、最 有效的手段之一[4]。

类石墨非晶碳膜(GLC) 具有优异的内在特性, 如 在去离子水和海水环境中较低的摩擦系数, 较高的 硬度和优异的电化学惰性等 ${ }^{[5-10]}$, 使其成为提高海洋 工程装备关键部件摩擦学性能的优选材料之一 ${ }^{[11]}$, 受到广泛关注。但是, 由于 GLC 与不锈钢等金属基 底结合力较低, 容易产生薄膜剥落, 从而限制其工业 应用 ${ }^{[12-13]}$ 。构筑金属过渡层提高膜基结合力可以解决 这一问题 ${ }^{[14-17]}$, 其中, 金属 $\mathrm{Cr}, \mathrm{Ti}, \mathrm{W}$ 过渡层被广泛应 用 ${ }^{[18]}$ 。例如, 王永欣等 ${ }^{[19]}$ 通过非平衡磁控溅射方法制 备 $\mathrm{Cr} / \mathrm{GLC}$ 薄膜, 该薄膜在去离子水中表现出优异的 摩擦学性能。

尽管通过添加金属过渡层可以有效改善类石墨非 晶碳膜的膜基结合力和力学、摩擦性能, 但其对 GLC 薄膜微结构的影响仍缺乏深入研究, 尤其因金属类型 不同而造成的金属层与碳层界面处非晶碳的微结构变 化及相应机理仍有待澄清。

本工作采用直流磁控溅射技术在单晶硅片(100) 和 316L 不锈钢基底上分别制备 Cr/GLC、 Ti/GLC 和 W/GLC 三种不同金属过渡层的 GLC 薄膜, 研究 Cr, Ti, $\mathrm{W}$ 过渡层对类石墨非晶碳膜微结构、机械性能以及海 水环境下摩擦性能的影响, 并重点通过电子能量损失 谱(EELS)检测技术剖析金属/碳层界面处非晶碳的微 结构变化, 及其对摩擦性能的作用机理。

\section{1 实验方法}

\section{1 薄膜制备}

使用直流磁控溅射技术制备不同金属过渡层的类 石墨非晶碳膜。基底材料分别为硅片和厚度为 $1 \mathrm{~mm}$ 的镜面抛光的 $316 \mathrm{~L}$ 不锈钢 $(3 \mathrm{~cm} \times 3 \mathrm{~cm})$ 。硅片基底样 品用于薄膜表面形貌 SEM, 截面 TEM 等微观结构表 征。不锈钢基底样品用于耐腐蚀性能和海水环境下摩 擦学性能的测试。首先将基底材料依次放置在无水酒 精和丙酮中超声清洗 $15 \mathrm{~min}$, 利用吹风机烘干后粘贴
在样品架上。抽真空至 $4 \times 10^{-3} \mathrm{~Pa}$ 时, 通入一定量的 $\mathrm{Ar}$ 气, 使腔体气压保持在 $1.0 \mathrm{~Pa}$, 设置基底偏压为 $-300 \mathrm{~V}$, 通过 $\mathrm{Ar}$ 离子辉光放电刻蚀基体 $30 \mathrm{~min}$ 。然后, 打开金属靶(纯度 99.99\%)直流溅射电源沉积金属过渡 层, 并通过更换靶材, 沉积 $\mathrm{Cr} 、 \mathrm{Ti} 、 \mathrm{~W}$ 三种过渡层。 在沉积过程中, 溅射电流为 $3.0 \mathrm{~A}$, 基底偏压为 $-100 \mathrm{~V}$, 为保证过渡层厚度相同沉积时间分别为 $10 、 5$ 和 5 min。 最后, 打开石墨靶(纯度 99.99\%)直流电源, 沉积 GLC 薄膜, 其中基底偏压为-200 $\mathrm{V}$, 浌射电流为 $3.0 \mathrm{~A}$, 沉 积时间为 $60 \mathrm{~min}$ 。在沉积过程中, $\mathrm{Ar}$ 气分压为 $0.28 \mathrm{~Pa}$, 样品架在溅射过程中自转以保持薄膜均匀性。

\section{2 表征与分析}

由 RENISHAW in via 型共聚焦拉曼(Raman)光谱 检测仪器分析 GLC 薄膜的微观结构, 波长为 $532 \mathrm{~nm}$ 。 采用 S-4800 场发射扫描电镜(FESEM)和 TF20 型高分 辨透射电子显微镜(HRTEM)观察薄膜微观形貌和结构, 并使用透射电镜的 EELS 模块对类石墨非晶碳膜微观 结构进行定量分析。使用纳米压痕仪(MTS NANO G200), 在连续刚度模式下表征薄膜的硬度和弹性模 量。使用 AUTOLAB 三电极系统在室温下对薄膜动电 位极化曲线和电化学阻抗谱进行测试, 铂电极为辅助 电极, 饱和甘采电极为参比电极, 沉积 GLC 薄膜的 $316 \mathrm{~L}$ 不锈钢基底为工作电极, 测试表面为 $1 \mathrm{~cm}^{2}$, 扫 描范围为 $-0.7 \sim 1.3 \mathrm{~V}$, 扫描速率为 $0.5 \mathrm{mV} / \mathrm{s}$, 腐蚀溶液 为人工海水, 依据标准 ASTM 1141-98 ${ }^{[20]}$ 配制, 海水 成分如表 1 所示。采用球盘式往复运动摩擦磨损试验 机(RTEC MFT5000)表征样品的摩擦学性能, 摩擦测 试条件为: 加载 $5 \mathrm{~N}$, 频率 $2 \mathrm{~Hz}$, 摩擦副 $\mathrm{Si}_{3} \mathrm{~N}_{4}(\phi 6 \mathrm{~mm})$, 磨痕长度为 $5 \mathrm{~mm}$, 摩擦时间为 $60 \mathrm{~min}$, 摩擦测试环境 为人工海水。Alpha-Step IQ 表面轮廓仪用来表征磨痕 深度剖面，并利用磨损率公式 ${ }^{[21]}$ 计算磨损率。

\section{2 结果与讨论}

\section{1 薄膜的微观形貌和微观结构}

图 1 为硅片上制备的不同金属过渡层 GLC 薄膜的 表面和截面形貌。由表面形貌可以得出, 由直流磁控 溅射制备的 GLC 薄膜表面平整, 没有大颗粒等明显缺 陷。通过截面 SEM 照片可知, Cr/GLC, Ti/GLC 和 W/GLC 三种薄膜的厚度为 $(600 \pm 50) \mathrm{nm}$, 其中 GLC 薄 膜厚度为 $(400 \pm 50) \mathrm{nm}$ 。由于金属 $\mathrm{Cr}$ 比 $\mathrm{Ti}, \mathrm{W}$ 的沉积速 率高, 过渡层 $\mathrm{Cr}$ 的厚度略大于 $\mathrm{Ti}$ 和 $\mathrm{W}$ 。

表 1 人工海水配方 $/\left(\mathrm{g} \cdot \mathrm{L}^{-\mathbf{1}}\right)$

Table 1 Chemical composition of artificial seawater/(g. $\left.\mathrm{L}^{-1}\right)$

\begin{tabular}{ccccccccccc}
\hline Constituent & $\mathrm{NaCl}$ & $\mathrm{MgCl}_{2}$ & $\mathrm{Na}_{2} \mathrm{SO}_{4}$ & $\mathrm{CaCl}_{2}$ & $\mathrm{KCl}$ & $\mathrm{NaHCO}_{3}$ & $\mathrm{KBr}$ & $\mathrm{H}_{3} \mathrm{BO}_{3}$ & $\mathrm{SrCl}_{2}$ & $\mathrm{NaF}$ \\
\hline Concentration & 24.530 & 5.200 & 4.090 & 1.160 & 0.695 & 0.201 & 0.101 & 0.027 & 0.025 & 0.003 \\
\hline
\end{tabular}


图 2 为硅片上制备的不同金属过渡层 GLC 薄膜的 高分辨透射电镜照片及对应的选区衍射结果。其中金 属 $\mathrm{Cr}$ 和 $\mathrm{Ti}$ 过渡层呈现典型的柱状晶生长, 在 $\mathrm{Cr} / \mathrm{GLC}$ 薄膜的铬碳界面可以观察到显著的界面“咬合”结构; W/GLC 薄膜除典型的铇碳双层结构外, 无明显特征。由 选区衍射结果可知, 制备的碳膜呈现典型的非晶结构。

拉曼光谱对碳材料的化学键结构和团簇尺寸等具 有优异的分辨能力, 且操作简单, 对薄膜材料没有破坏 性，是非晶碳膜常用的分析测试方法之一。典型非晶碳 膜的拉曼光谱经过高斯拟合之后在 $1350 \mathrm{~cm}^{-1}$ 出现 D 峰, 在 $1560 \mathrm{~cm}^{-1}$ 处出现 $\mathrm{G}$ 峰, 两峰均对应非晶碳膜 $\mathrm{sp}^{2}$ 相
应的振动模式 ${ }^{[22]}$ 。图 3 为不锈钢基底上三种薄膜的 $I_{\mathrm{D}} / I_{\mathrm{G}} 、 \mathrm{G}$ 峰、G $G_{\mathrm{FWHM}}, \mathrm{W} / \mathrm{GLC}$ 薄膜 $I_{\mathrm{D}} / I_{\mathrm{G}}$ 值为 $1.48, \mathrm{G}$ 峰 位置为 $1545.3 \mathrm{~cm}^{-1}, G_{\mathrm{FWHM}}$ 值为 $158.9 \mathrm{~cm}^{-1}$ 。对于不含 氢的非晶碳膜而言, $I_{\mathrm{D}} I_{\mathrm{G}}$ 值越大, 非晶碳膜中的 $\mathrm{sp}^{2}$ 杂化 键含量越多。由图 3 可知, 相比较于 W/GLC, Ti/GLC 和 $\mathrm{Cr} / \mathrm{GLC}$ 的 $I_{\mathrm{D}} / I_{\mathrm{G}}$ 值增大, 分别为 2.78 和 3.0; $\mathrm{G}$ 峰位置增 大到 1551.2 和 $1554.7 \mathrm{~cm}^{-1}$; $\mathrm{G}_{\mathrm{FWHM}}$ 分别降低至 156.5 和 $155.2 \mathrm{~cm}^{-1}$ 。由此可得出, 不同金属过渡层对类石墨非 晶碳薄膜微结构影响不同, $\mathrm{Cr} / \mathrm{GLC}$ 具有最多的 $\mathrm{sp}^{2}$ 杂化 键含量。

为了进一步探究这一影响, 采用电子能量损失谱
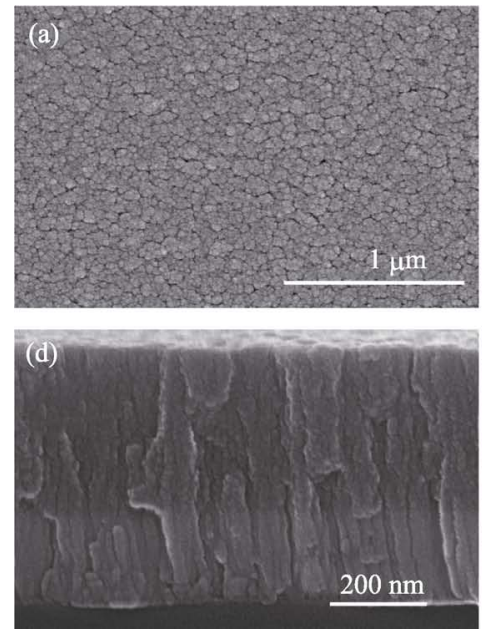
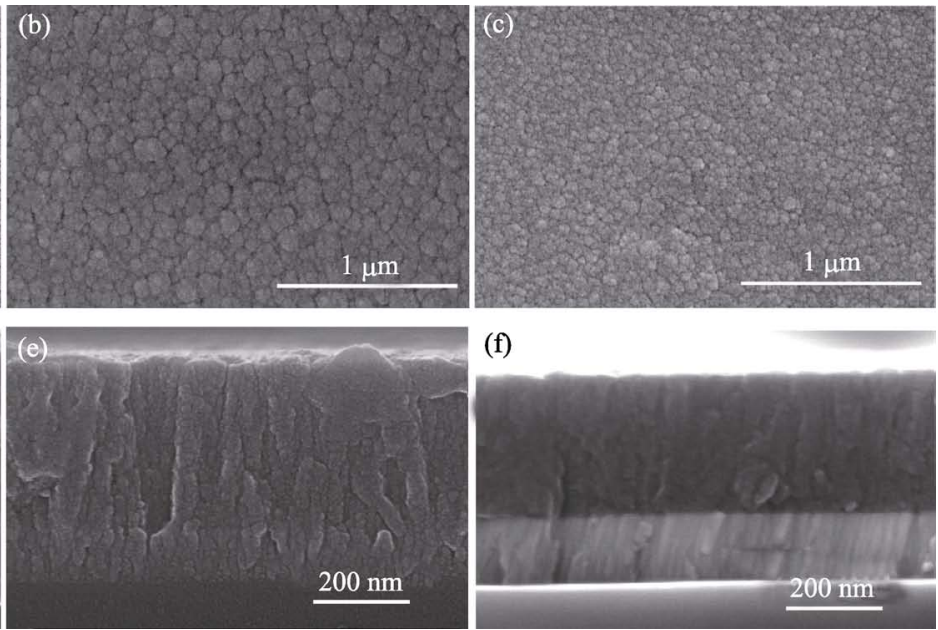

(f)

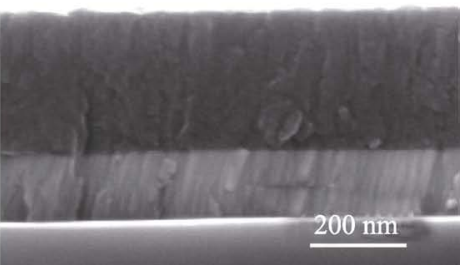

图 1 不同金属过渡层 GLC 薄膜的表面和截面 SEM 照片

Fig. 1 Surface and cross-sectional morphologies of GLC films with different metal buffer layers (a, d) $\mathrm{Cr} / \mathrm{GLC}$; (b, e) Ti/GLC; (c, f) W/GLC
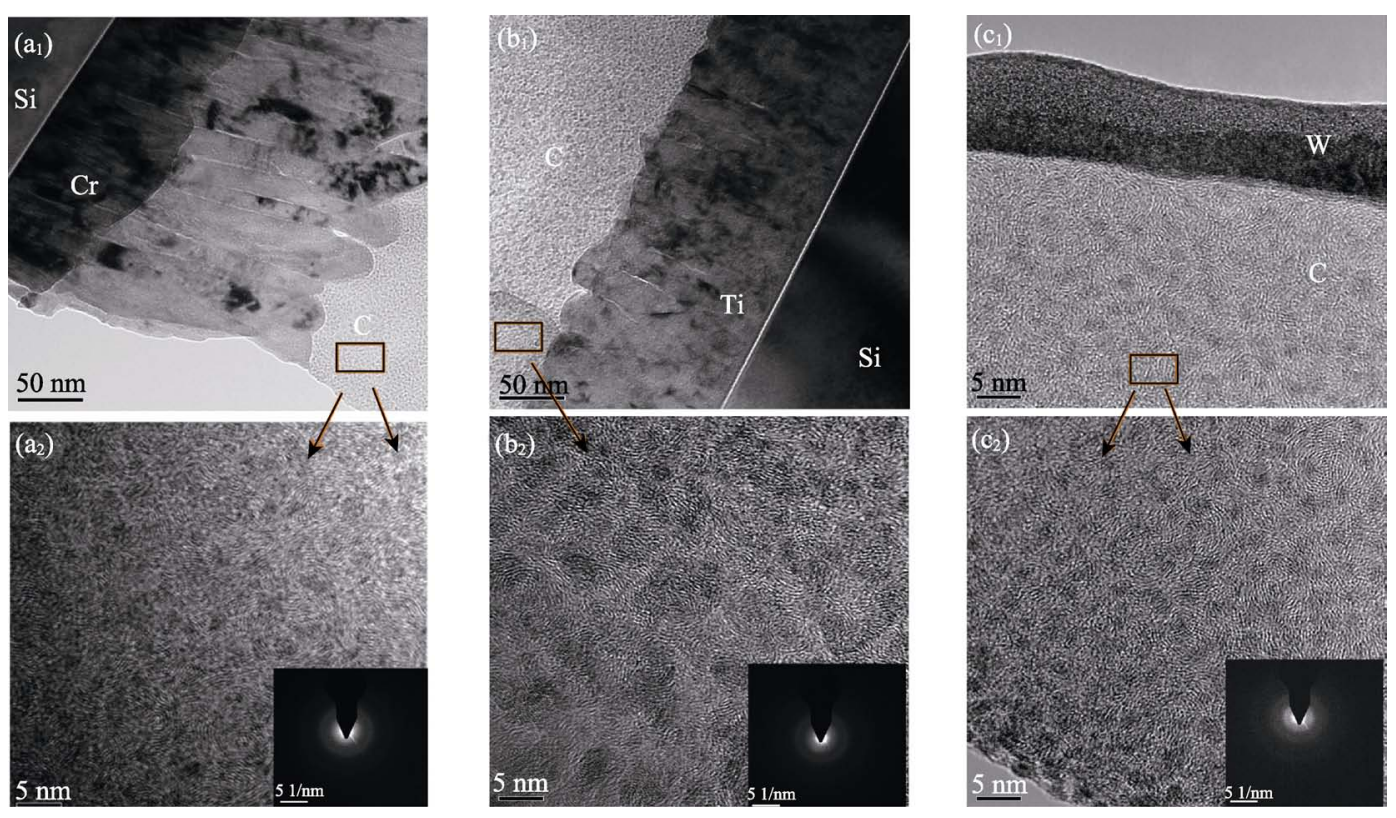

图 2 不同金属过渡层 GLC 薄膜的截面 HRTEM 照片及对应的非晶碳层的 SAED 图案

Fig. 2 Cross-sectional high-resolution transmission electron microscopy (HRTEM) images and SAED patterns of the GLC films with different metal buffer layers

(a) $\mathrm{Cr} / \mathrm{GLC}$; (b) Ti/GLC; (c) W/GLC 

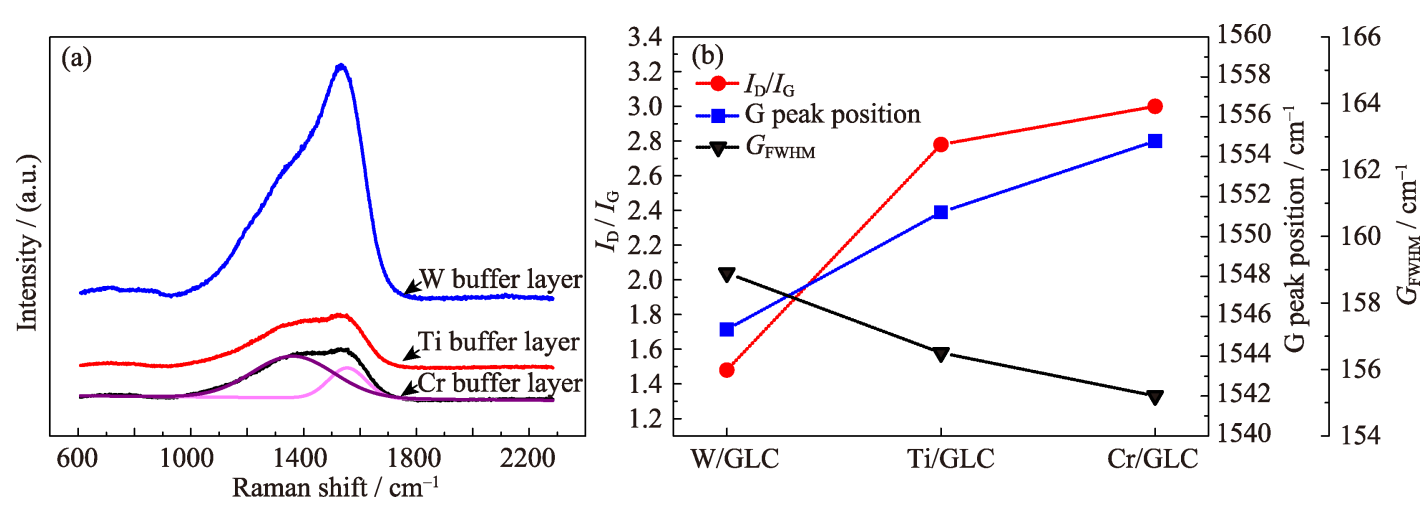

图 3 不同金属过渡层 GLC 薄膜的(a)拉曼光谱, (b)G 峰半峰宽和 $\mathrm{G}$ 峰位置 $I_{\mathrm{D}} / I_{\mathrm{G}}$

Fig. 3 (a) Raman spectra and (b) fitted $I_{\mathrm{D}} / I_{\mathrm{G}}$, G peak position, $G_{\mathrm{FWHM}}$ of the GLC films with different metal buffer layers
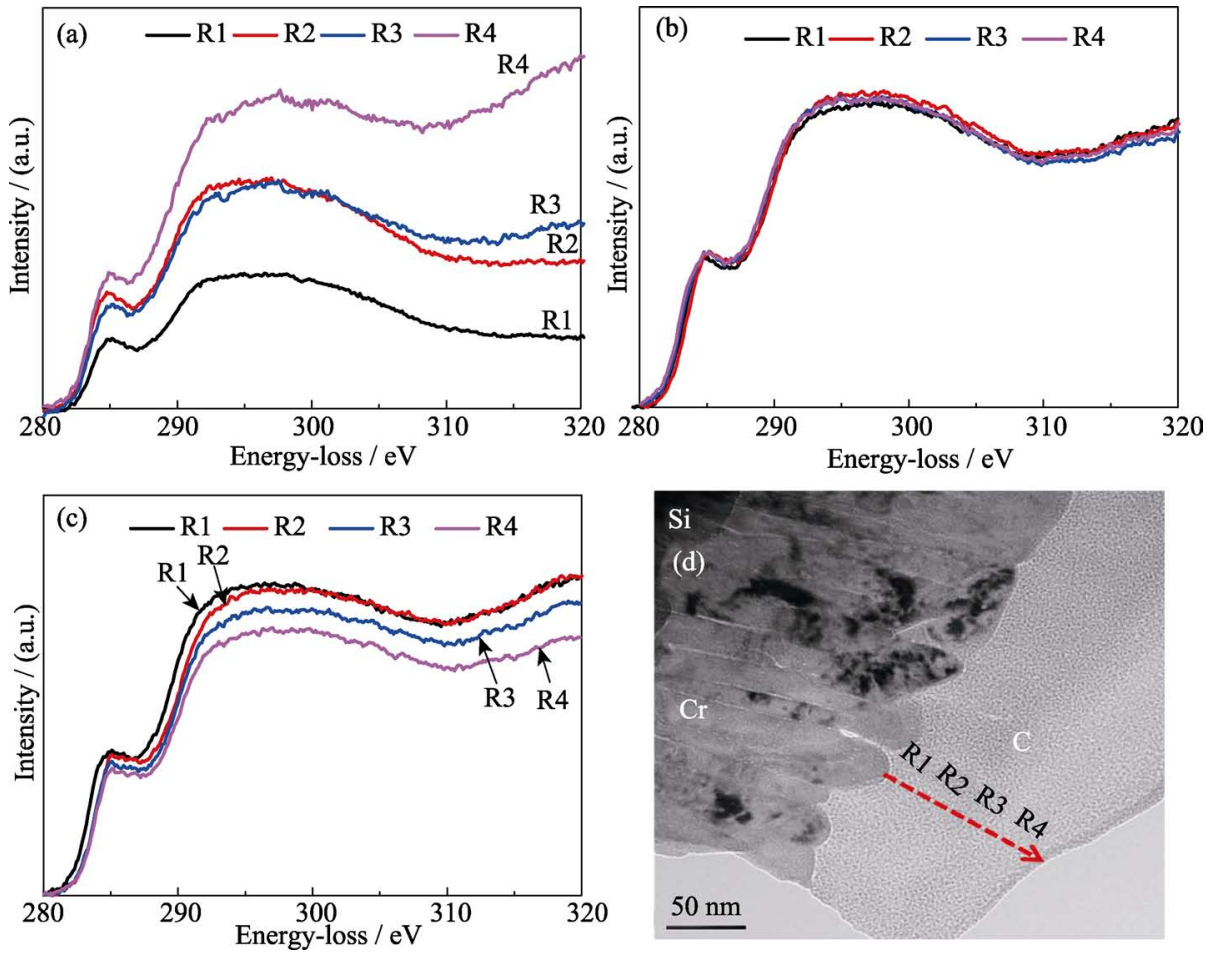

图 4 不同金属过渡层 GLC 薄膜的电子能量损失谱(EELS)

Fig. 4 EELS spectra of the GLC films with different metal buffer layers (a) Cr/GLC; (b) Ti/GLC; (c) W/GLC; (d) Position schematic diagram of taking EELS spectra

(EELS)对界面进行深入分析。图 4 为不同金属过渡层 的 GLC 薄膜“金属一非晶碳层”界面处的 EELS 结果。 图 4 为硅片上制备的不同金属过渡层 GLC 薄膜“金属非晶碳层” 界面处的 EELS 结果。各薄膜的四个探测位 置如图 4(d)所示, 从界面处至表面分别标定为: R1, R2, R3, R4。高定向裂解石墨(HOPG)作为标样。通过“双 窗口法”, ${ }^{[23-25]}$ 拟合计算, $\mathrm{sp}^{2}$ 杂化键含量由样品 $I_{\pi^{*}} / I_{\sigma^{*}}$ 与 标样 $I_{\pi^{*}} / I_{\sigma^{*}}$ (HOPG sp 杂化键含量为 100\%)的比例确 定。 $I_{\sigma^{*}}$ 的积分区间优化为 $292 \sim 308 \mathrm{eV}, I_{\pi^{*}}$ 的积分区间 为 $284.7 \sim 285.3 \mathrm{eV}$ 。经过计算及与标样 HOPG 对比, 不 同金属过渡层 GLC 薄膜中 $\mathrm{R} 1$ 至 $\mathrm{R} 4$ 的 $\mathrm{sp}^{2}$ 杂化键含量 为: $(0.62 \pm 0.034) 、(0.56 \pm 0.033) 、(0.50 \pm 0.037) 、(0.44 \pm$ $0.045)$ (图 4(a)); $(0.51 \pm 0.048) 、(0.50 \pm 0.040) 、(0.49 \pm$

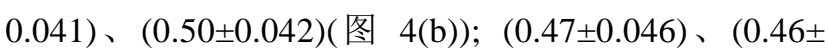
$0.049) 、(0.47 \pm 0.049) 、(0.47 \pm 0.047)$ (图 4(c))。Cr/GLC 薄 膜从界面处至表面, 非晶碳层中 $\mathrm{sp}^{2}$ 杂化键含量逐渐 降低，铬碳界面特殊的“咬合”结构促进了界面处的石 墨化转变, 从而提高薄膜整体的 $\mathrm{sp}^{2}$ 杂化键含量。

\section{2 薄膜的机械性能}

图 5 为 316L 不锈钢上制备的不同金属过渡层 GLC 薄膜的硬度和弹性模量, Cr/GLC 的硬度和弹性模量最 低: (20.22 \pm 1.74$) \mathrm{GPa},(230.12 \pm 19.56) \mathrm{GPa}$; W/GLC 薄膜 的硬度最高。这是因为薄膜硬度与 $\mathrm{sp}^{3}$ 杂化键含量相关, 高 $\mathrm{sp}^{3} \mathrm{C}-\mathrm{C}$ 含量会导致硬度增加 ${ }^{[26]}$, 而由图 3 可知, $\mathrm{Cr} / \mathrm{GLC}$ 薄膜中 $\mathrm{sp}^{3}$ 含量最低, 石墨化程度的增高降低 了薄膜的硬度。 


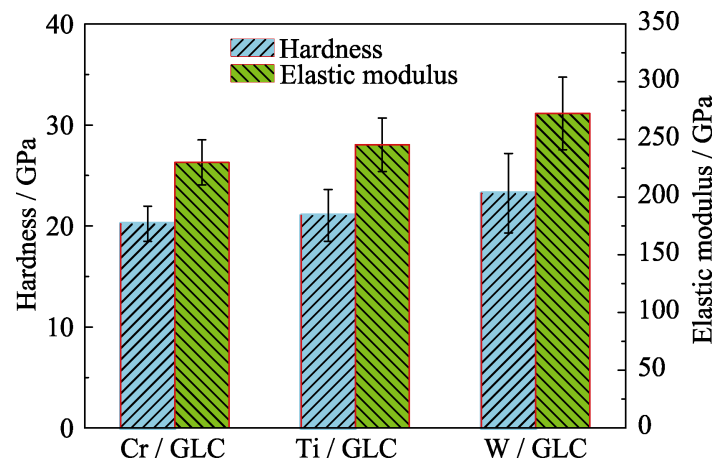

图 5 不同金属过渡层 GLC 薄膜的机械性能

Fig. 5 Mechanical properties of the GLC films with different metal buffer layers

\section{3 薄膜的电化学性能}

海水溶液中的极化测试结果如图 6 所示。316L 不锈钢基底在海水环境下耐蚀性较差，腐蚀电位为 $-0.32 \mathrm{~V}$, 腐蚀电流密度为 $1.45 \times 10^{-5} \mathrm{~A} / \mathrm{cm}^{2}$ 。制备不同 金属过渡层的 GLC 薄膜将 316L 不锈钢基底的腐蚀电 位提高约 $0.2 \mathrm{~V}$, 腐蚀电流密度降低 3 4 个数量级。 $\mathrm{Cr} / \mathrm{GLC}$ 薄膜的自腐蚀电位和腐蚀电流密度分别为 $-0.16 \mathrm{~V}$ 和 $4.42 \times 10^{-9} \mathrm{~A} / \mathrm{cm}^{2}$, 表现出最好的耐蚀性能, 这能得益于其特殊的层间“咬合”结构, 紧密的咬合结 构阻碍了腐蚀过程中缺陷的扩展和腐蚀通道的形成。 $\mathrm{Ti} / \mathrm{GLC}$ 薄膜耐蚀性次之, 其腐蚀电位为 $-0.17 \mathrm{~V}$, 腐蚀电 流密度为 $1.28 \times 10^{-8} \mathrm{~A} / \mathrm{cm}^{2}$ 。W/GLC 薄膜耐蚀性最差, 其 腐蚀电位为 $-0.22 \mathrm{~V}$, 腐蚀电流密度为 $3.28 \times 10^{-8} \mathrm{~A} / \mathrm{cm}^{2}$ 。

图 7 为三种 $\mathrm{Me} / \mathrm{GLC}$ 薄膜在人工海水中的电化学 交流阻抗图谱及相应的等效电路。等效电路中, $R_{\mathrm{s}}$ 为试 样与参比电极之间的溶液电阻; $Q_{1}$ 代表三种 Me/GLC 薄 膜的电容, $R_{1}$ 为离子通过薄膜孔隙传导的电阻, $Q_{2}$ 代表

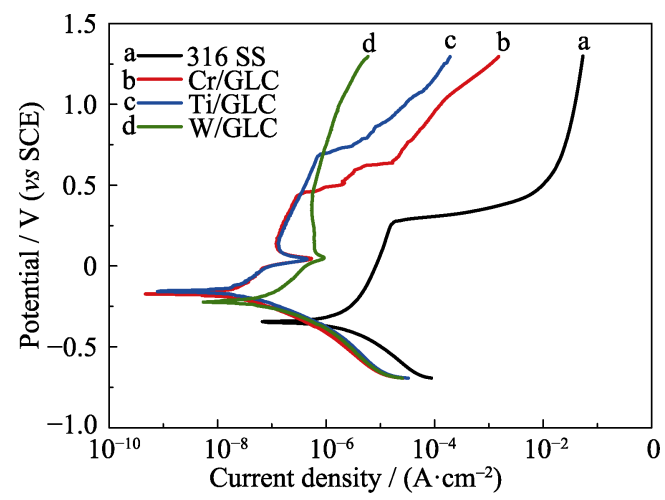

图 $6316 \mathrm{~L}$ 不锈钢基底与不同金属过渡层 GLC 薄膜在人工 海水中的动电位极化曲线

Fig. 6 Potentiodynamic polarization curves of the 316LSS substrate and GLC films with different metal buffer layers in artificial seawater

溶液到达薄膜/基体界面产生的双电层电容, $R_{2}$ 为相应 的传质电阻。Nyquist 图中, $\mathrm{Cr} / \mathrm{GLC}$ 薄膜呈现最大半径 的容抗弧, 表明在三种 $\mathrm{Me} / \mathrm{GLC}$ 薄膜中, $\mathrm{Cr} / \mathrm{GLC}$ 的腐蚀 抗力明显高于 Ti/GLC 和 W/GLC。Bode 图中, 相角在 不同频率区存在两个峰值, 意味着试样表面的电化学 过程含两个时间常数, 其中高频区反映了薄膜的腐蚀 行为, 低频区则代表薄膜/基体界面的电化学过程。从图 7(a)和(b)中可以看出, 拟合结果与阻抗测试结果高度重 合, 说明采用的等效电路拟合分析很好地反映了三种 $\mathrm{Me} / \mathrm{GLC}$ 薄膜在人工海水中的电化学腐蚀行为。表 2 为 相应的拟合结果, 可以发现 $\mathrm{Cr} / \mathrm{GLC}$ 薄膜具有最高的极 化电阻 $R_{\mathrm{p}}\left(R_{\mathrm{p}}=R_{1}+R_{2}\right) \quad 1.244 \times 10^{6} \Omega \cdot \mathrm{cm}^{2}$, 反映出最大的 腐蚀抗力。此外, $\mathrm{Cr} / \mathrm{GLC}$ 薄膜的孔隙电阻 $R_{1}$ 比 Ti/GLC 和 W/GLC 均高一个数量级, 说明 $\mathrm{Cr} / \mathrm{C}$ 界面间的特殊咬 合结构阻碍了缺陷的扩展和腐蚀通道的形成, 抑制了
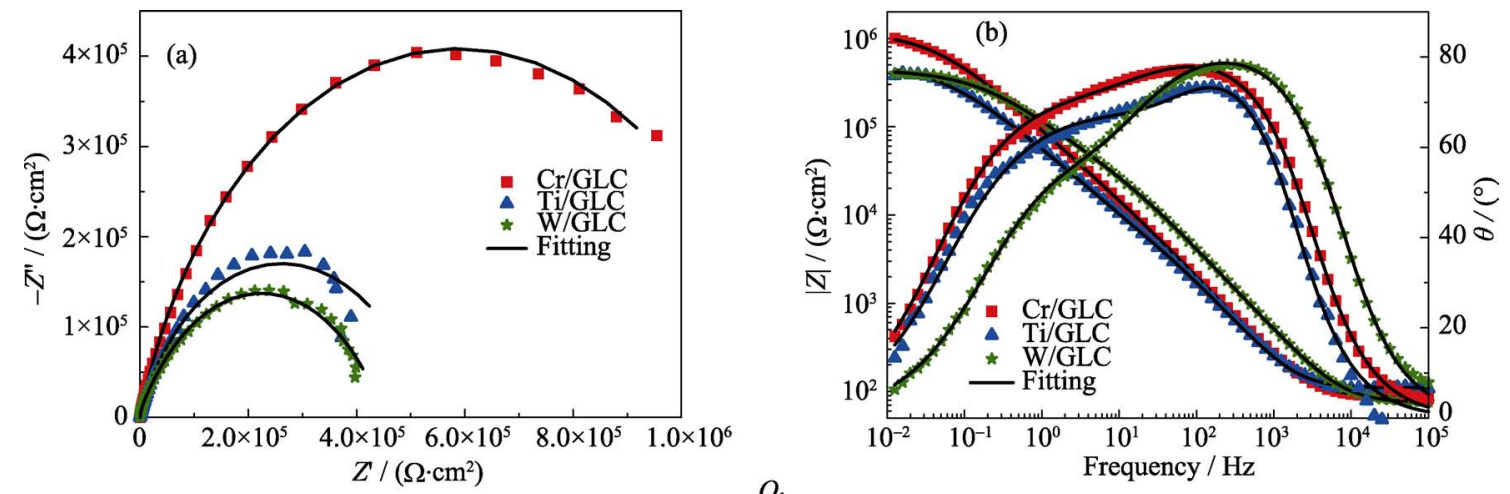

$Q_{1}$

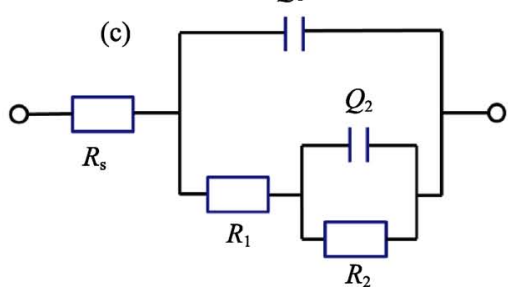

图 7 不同金属过渡层 GLC 薄膜在人工海水环境下的电化学阻抗图谱

Fig. 7 EIS results of the GLC films with different metal buffer layers in artificial seawater

(a) Nyquist plots; (b) Bode plots; (c) Equivalent circuit 
表 2 不同金属过渡层 GLC 薄膜的 EIS 拟合数据

Table 2 Fitting data of EIS results for the GLC films with different metal buffer layers

\begin{tabular}{cccccccc}
\hline Sample & $R_{\mathrm{S}} /\left(\Omega \cdot \mathrm{cm}^{2}\right)$ & $Q_{1} /\left(\Omega^{-1} \cdot \mathrm{cm}^{-2} \cdot \mathrm{s}^{n}\right)$ & $n_{1}$ & $R_{1} /\left(\Omega \cdot \mathrm{cm}^{2}\right)$ & $Q_{2} /\left(\Omega^{-1} \cdot \mathrm{cm}^{-2} \cdot \mathrm{s}^{n}\right)$ & $n_{2}$ & $R_{2} /\left(\Omega \cdot \mathrm{cm}^{2}\right)$ \\
\hline Cr/GLC & 85.1 & $1.3 \times 10^{-6}$ & 0.91 & $4.4 \times 10^{4}$ & $1.4 \times 10^{-6}$ & 0.64 & $1.2 \times 10^{6}$ \\
Ti/GLC & 110.1 & $1.2 \times 10^{-6}$ & 0.94 & $7.3 \times 10^{3}$ & $3.4 \times 10^{-6}$ & 0.66 & $5.3 \times 10^{5}$ \\
W/GLC & 74.9 & $6.2 \times 10^{-7}$ & 0.92 & $4.4 \times 10^{3}$ & $1.9 \times 10^{-6}$ & 0.64 & $4.1 \times 10^{5}$ \\
\hline
\end{tabular}

腐蚀性溶液在涂层内部的扩散渗透, 从而赋予 $\mathrm{Cr} / \mathrm{GLC}$ 薄膜高的腐蚀抗力。

\section{4 薄膜的摩擦磨损性能}

图 8(a)所示为 316L 不锈钢上制备的不同金属过 渡层的 GLC 薄膜在人工海水环境中的摩擦测试结果。 $\mathrm{Cr} / \mathrm{GLC}$ 薄膜在前 $500 \mathrm{~s}$ 略有波动, 之后进入稳定阶段, 摩擦系数曲线平稳, 表现出优异的润滑特性。而 Ti/GLC 薄膜在 $1250 \mathrm{~s}$ 以后摩擦系数上升, 开始进入稳 定阶段。W/GLC 薄膜在经过磨合阶段之后, 摩擦系数 迅速增大, $500 \mathrm{~s}$ 后薄膜被磨穿。图 8(b)为薄膜稳定阶 段的平均摩擦系数和平均磨损率, 从图中可以看出, $\mathrm{Cr} / \mathrm{GLC}$ 薄膜在人工海水条件下表现出最低的摩擦系 数和磨损率: $(0.08 \pm 0.006)$ 和 $(2.3 \pm 0.3) \times 10^{-8} \mathrm{~mm}^{3} / \mathrm{Nm}$ 。

图 9 为三种薄膜磨痕的 SEM 照片, 如图所示, W/GLC 薄膜发生显著剥落, 薄膜被磨穿。在滑动过程 中, 海水通过薄膜本体缺陷, 如针孔等进入薄膜。在静
水楔入的物理作用和人工海水高浓度 $\mathrm{Cl}^{-}$电化学腐蚀 作用的共同影响下, 薄膜本体缺陷进一步扩大, 形成 腐蚀通道。在通道周围的薄膜材料或基底材料与海水 介质发生电化学反应, 造成材料损失 ${ }^{[27]}$ 。因此, 在海水 条件下, 由于薄膜缺陷扩展所造成的薄膜本体剥落, 是 W/GLC 薄膜失效的主要原因。从 Ti/GLC 和 Cr/GLC 薄膜的磨痕形貌中, 同样可以观察到薄膜的局部剥 落。相比较于 $\mathrm{Ti} / \mathrm{GLC}$ 薄膜, $\mathrm{Cr} / \mathrm{GLC}$ 薄膜的磨痕局部 剥落程度更低, 摩擦学性能更优异。

为了进一步剖析 $\mathrm{Cr} / \mathrm{GLC}$ 薄膜摩擦学性能优异的 原因, 对对偶球磨斑进行 Raman 光谱分析(图 10)。 $\mathrm{Cr} / \mathrm{GLC}$ 薄膜磨斑在 $1580 \mathrm{~cm}^{-1}$ 处出现非晶碳的特征峰, 说明在摩擦过程中有含碳转移膜在对偶球上形成。 Ti/GLC 薄膜对偶球上转移膜石墨化的特征峰不明显。 W/GLC 薄膜对偶球上无石墨化特征峰。这是由于 $\mathrm{Cr} / \mathrm{GLC}$ 薄膜中较高的 $\mathrm{sp}^{2}$ 含量有助于摩擦过程中石墨
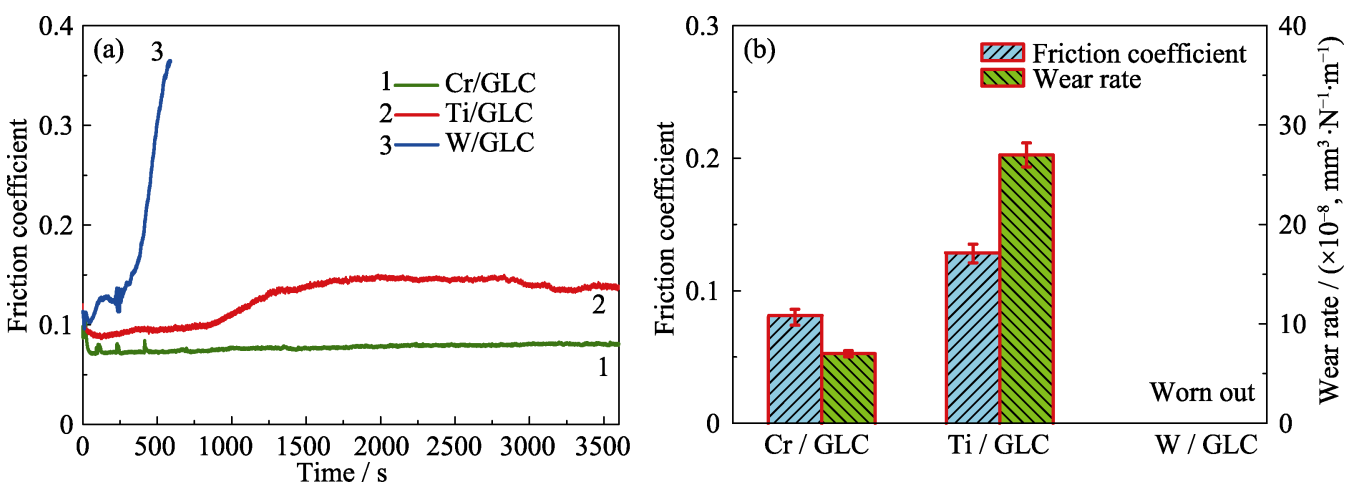

图 8 不同金属过渡层 GLC 薄膜在人工海水条件下的摩擦曲线(a)和平均摩擦系数和磨损率(b)

Fig. 8 (a) Friction coefficient curves of the GLC films with different metal buffer layers in artificial seawater and (b) average friction coefficients and wear rates of GLC films with different metal buffer layers
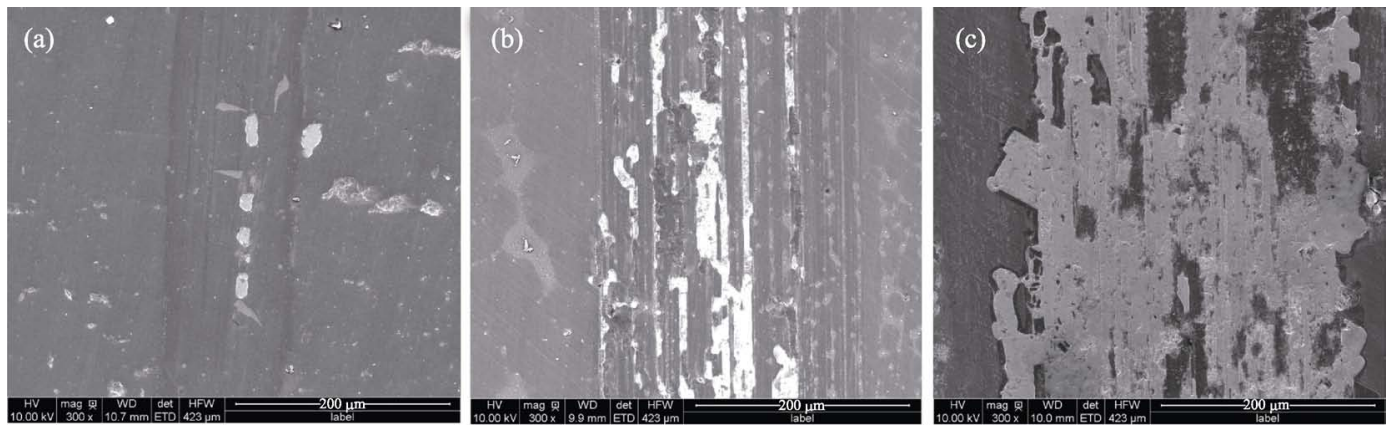

图 9 不同薄膜在海水环境下磨痕的 SEM 照片

Fig. 9 SEM images of wear track of the GLC films in artificial seawater

(a) $\mathrm{Cr} / \mathrm{GLC}$; (b) Ti/GLC; (c) W/GLC 

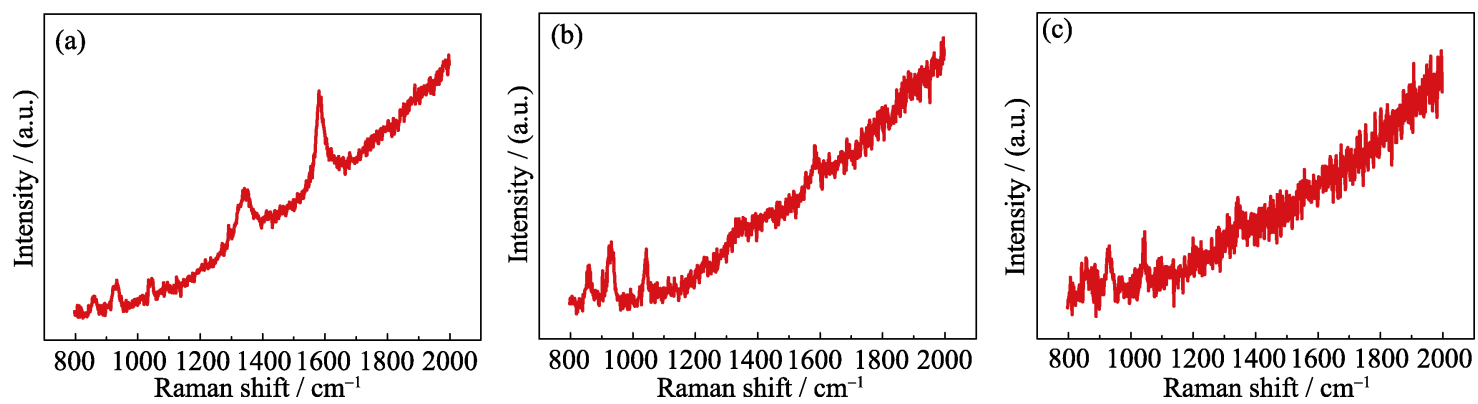

图 10 不同金属过渡层 GLC 薄膜 $\mathrm{Si}_{3} \mathrm{~N}_{4}$ 对偶球磨斑表面的拉曼光谱

Fig. 10 Raman spectra on wear scars of $\mathrm{Si}_{3} \mathrm{~N}_{4}$ balls for the GLC films (a) Cr/GLC; (b) Ti/GLC; (c) W/GLC

化转移膜的形成, 从而显著降低摩擦系数和磨损率。 综上, 优异的耐蚀性和摩擦过程中转移膜的石墨化转 化是 $\mathrm{Cr} / \mathrm{GLC}$ 薄膜在海水条件下摩擦学性能优异的主 要原因。

\section{3 结论}

1) TEM 照片显示, 不同金属过渡层界面结构不 同。三种体系中, Cr/GLC 薄膜在界面处具有较为明显的 “咬合”结构。Raman 和 EELS 结果显示, “咬合”结构促 进了界面处的石墨化转变, 从而提高薄膜整体 $\mathrm{sp}^{2}$ 杂化 键含量。但是较高的 $\mathrm{sp}^{2}$ 杂化键含量对薄膜硬度也造成 了一定程度的损失;

2) Cr/GLC 薄膜界面处紧密的“咬合”结构阻碍了腐 蚀过程中缺陷的扩展和腐蚀通道的形成, 从而使 $\mathrm{Cr} / \mathrm{GLC}$ 薄膜具有优异的耐蚀性：腐蚀电位为 $-0.16 \mathrm{~V}$, 腐蚀电流密度为 $4.42 \times 10^{-9} \mathrm{~A} / \mathrm{cm}^{2}$ 。将不锈钢基底的腐蚀 电位提高 $0.2 \mathrm{~V}$, 腐蚀电流密度降低 4 个数量级, 耐蚀性 优于 Ti/GLC 和 W/GLC 薄膜;

3) $\mathrm{Cr} / \mathrm{GLC}$ 薄膜较高的 $\mathrm{sp}^{2}$ 杂化键含量有助于其在 摩擦过程中产生石墨化转化的摩擦转移膜。在对偶球上 形成的石墨化摩擦转移膜可以在摩擦过程中充当润滑 相, 并显著降低摩擦系数和磨损率。在优异的耐蚀性和 石墨化摩擦转移膜的共同作用下, 相较于 Ti/GLC 和 W/GLC 薄, Cr/GLC 薄膜在人工海水中表现出优异的摩 擦学性能: 摩擦系数和磨损率分别为 $(0.08 \pm 0.006)$ 和 $(2.3 \pm 0.3) \times 10^{-8} \mathrm{~mm}^{3} / \mathrm{Nm}$ 。

\section{参考文献:}

[1] YANG SHU-DONG, LI ZHUANG-YUN. Seawater hydraulic drive and its application in ocean exploitation. Ocean Engineering, 2000, 18(1): 81-85.

[2] COSTA R P C, LIMA-OLIVEIRA D A, MARCIANO F R, et al. Comparative study of the tribological behavior under hybrid lubrication of diamond-like carbon films with different adhesion interfaces. Applied Surface Science, 2013, 285P(11): 645-648.

[3] HIRANI H, GOILKAR S S. Formation of transfer layer and its effect on friction and wear of carbon-graphite face seal under dry, water and steam environments. Wear, 2009, 266(11): 1141-1154.

[4] WOOD R J K. Erosion-corrosion interactions and their effect on marine and offshore materials. Wear, 2006, 261(9): 1012-1023.

[5] GAHR K H Z, MATHIEU M, BRYLKA B. Friction control by surface engineering of ceramic sliding pairs in water. Wear, 2007, 263(7-12): 920-929.

[6] GILEWICZ A, WARCHOLINSKI B, MYSLINSKI P, et al. Anti-wear multilayer coatings based on chromium nitride for wood machining tools. Wear, 2010, 270(1): 32-38.

[7] HUANG M, ZHANG X, KE P, et al. Graphite-like carbon films by high power impulse magnetron sputtering. Applied Surface Science, 2013, 283(10): 321-326.

[8] KNOTEK O, L FFLER F, KR MER G. Multicomponent and multilayer physically vapour deposited coatings for cutting tools. Surface \& Coatings Technology, 1992, 54-55(Part 1): 241-248.

[9] TAKADOUM J, HOUMID-BENNANI H, MAIREY D, et al. Adhesion and wear resistance of thin hard coatings. Journal of the European Ceramic Society, 1997, 17(15): 1929-1932.

[10] ZHOU F, YUAN Y, WANG X, et al. Influence of nitrogen ion implantation fluences on surface structure and tribological properties of SiC ceramics in water-lubrication. Applied Surface Science, 2009, 255(9): 5079-5087.

[11] TOTOLIN V, PEJAKOVIĆ V, CSANYI T, et al. Surface engineering of Ti6Al4V surfaces for enhanced tribocorrosion performance in artificial seawater. Materials \& Design, 2016, 104: $10-18$.

[12] VOEVODIN A A, DONLEY M S, ZABINSKI J S, et al. Mechanical and tribological properties of diamond-like carbon coatings prepared by pulsed laser deposition. Surface \& Coatings Technology, 1995, 76: 534-539.

[13] WANG Y, LI H, JI L, et al. Synthesis and characterization of titanium-containing graphite-like carbon films with low internal stress and superior tribological properties. Journal of Physics $D$ Applied Physics, 2012, 45(29): 556-563.

[14] CUI M, PU J, LIANG J, et al. Corrosion and tribocorrosion performance of multilayer diamond-like carbon film in $\mathrm{NaCl}$ solution. RSC Advances, 2015, 5(127): 104829-104840.

[15] HE X, LI W, LI H. Diamond - like carbon film synthesized by ion beam assisted deposition and its tribological properties. Journal of Vacuum Science \& Technology A Vacuum Surfaces \& Films, 1996, 14(4): 2039-2047.

[16] KIURU M, ALAKOSKI E, TIAINEN V M, et al. Tantalum as a buffer layer in diamond-like carbon coated artificial hip joints. Journal of Biomedical Materials Research Part B Applied Biomaterials, 2003, 66B(1): 425-428.

[17] WANG Y, WANG L, XUE Q. Controlling wear failure of graphite-like carbon film in aqueous environment: two feasible 
approaches. Applied Surface Science, 2011, 257(9): 4370-4376.

[18] LI Y S, TANG Y, YANG Q, et al. Growth and adhesion failure of diamond thin films deposited on stainless steel with ultra-thin dual metal interlayers. Applied Surface Science, 2010, 256(24): 76537657.

[19] GUAN X, LU Z, WANG L. Achieving high tribological performance of graphite-like carbon coatings on Ti6Al4V in aqueous environments by gradient interface design. Tribology Letters, 2011, 44(3): 315-325.

[20] WANG J Z, YAN F Y, XUE Q J. Tribological behaviors of some polymeric materials in seawater. Science Bulletin, 2009, 54(24): 4541-4548.

[21] QI J, WANG L, WANG Y, et al. The tribological performance of selected solid lubricant films in sand-dust environments. Wear, 2011, 271(5/6): 899-910.

[22] CHEN X, PENG Z, YU X, et al. Microstructure and tribological performance of self-lubricating diamond/tetrahedral amorphous carbon composite film. Applied Surface Science, 2011, 257(8): 3180-3186.
[23] BERGER S D, MCKENZIE D R, MARTIN P J. EELS analysis of vacuum arc-deposited diamond-like films. Philosophical Magazine Letters, 1988, 57(6): 285-290.

[24] BRULEY J, WILLIAMS D B, CUOMO J J, et al. Quantitative near-edge structure analysis of diamond-like carbon in the electron microscope using a two-window method. Journal of Microscopy, 2011, 180(180): 22-32.

[25] CUOMO J J, DOYLE J P, BRULEY J, et al. Sputter deposition of dense diamond-like carbon films at low temperature. Applied Physics Letters, 1991, 58(5): 466-468.

[26] ZHANG X, HUANG Q, LIU M, et al. Preparation of amine functionalized carbon nanotubes via a bioinspired strategy and their application in $\mathrm{Cu}^{2+}$ removal. Applied Surface Science, 2015, 343: 19-27.

[27] GAO S, DONG C, HONG L, et al. Scanning electrochemical microscopy study on the electrochemical behavior of $\mathrm{CrN}$ film formed on 304 stainless steel by magnetron sputtering. Electrochimica Acta, 2013, 114: 233-241. 